\title{
GESTIÓN CULTURAL Y DESPOLITIZACIÓN: cuando nos llamaron gestores
}

\author{
Paola De la Vega Velastegui
}

Fecha de recepción: 08/2016

Fecha de aceptación: 10/2016

\begin{abstract}
Resumen:
Este artículo permite una aproximación crítica al surgimiento de la gestión cultural en Ecuador y América Latina, como campo de conocimiento emergente en la década de los noventa, en un contexto de aplicación de agendas de ayuda al desarrollo, políticas neoliberales y de ajuste estructural, multiculturalismo incluyente, y la globalización expresada en la apertura nacional y la integración regional. Situada en estas complejas relaciones geopolíticas, la gestión cultural es tecnología de gobierno de la conducta (Richard, 2010, 179) y una estrategia de despolitización de prácticas artísticas y culturales de dimensión comunitaria y política con vigencia plena en este decenio. Propuestas artísticas y comunidades, dialógicas y de contexto, epistemologías de la diferencia, activismo, educación y comunicación popular, son algunos de los planos fundamentales de las discusiones académicas actuales, con perspectiva histórica, sobre una epistemología de la gestión cultural latinoamericana.
\end{abstract}

Palabras claves: gestión cultural, tecnología de gobierno, conocimiento situado, tecnologías sociales, prácticas contextualizadas, epistemología de la gestión cultural latinoamericana.

\begin{abstract}
:
This article provides a critical approach to the rise of cultural management in Ecuador and Latin America as a developing field of knowledge in 90s, in the context of development aid programs, neoliberal policies and structural adjustments, including multiculturalism and globalization, in national aperture and regional integration. In these complex geopolitical situations, cultural management stands like a skill to power the organization (Richard, 2010, 1979) and a strategy for depoliticization of artistic and cultural practices with a communitarian basis, valid within these ten years. Some approaches of academic debate, with historical perspective based on a LatinAmerican's cultural management epistemology are artistic projects, dialogical communities and their contexts, difference epistemology, activism, education, and communication.
\end{abstract}

Keywords: cultural management, government technology, situated knowledge, social technologies, contextualized practices, epistemology of Latin American cultural management.

\section{Autora:}

Paola de la Vega V. Gestora cultural, investigadora y docente universitaria. Doctoranda en Estudios Culturales Latinoamericanos por la Universidad Andina Simón Bolívar Sede Ecuador- UASB. Máster en Gestión Cultural por la Universidad Carlos III de Madrid. Lcda. en Comunicación con mención en Comunicación y Literatura por la Pontificia Universidad Católica del Ecuador-PUCE. Desde 2007 forma parte del colectivo Gescultura. Es docente de Investigación y Gestión Cultural en la Carrera de Artes Visuales de la PUCE; de Gestión de Museos en la Especialización de Museos y Patrimonio Histórico de la UASB; y de Políticas e Industrias Culturales en la Maestría de Estudios de la Cultura de la UASB. 
Si el imperativo de gestionar la cultura se volvió dominante en Ecuador, especialmente en el transcurso de la década de los noventa, esto no quiere decir que sus fundamentos asociados al emprendimiento, la planificación, la eficacia, la eficiencia o la sostenibilidad hayan producido un borramiento absoluto de prácticas contextualizadas de acción cultural. Stuart Hall $(2011,30)$, a partir de sus lecturas de Derrida, afirma que los conceptos no tienen significados predeterminados desde su comienzo; sin embargo, no queda más remedio que usarlos aunque no sean los más adecuados- porque no tenemos otro modo de hablar acerca de lo que éstos representan. De ahí que Hall (2011) invite a "pensar en los límites". Los conceptos, además, son coyunturales, se traducen en contextos específicos para abordar situaciones particulares; tienen trayectorias, se resignifican. Este es el primer paso para comprender que la gestión cultural, y con ella de las artes, tiene, por un lado, un corpus de ideas que constituye su hegemonía, y por otro, de acuerdo a sus usos sociales, especificidades locales, históricas, de posiciones frente a sus prácticas y discursos, ha adquirido significados diversos; por tanto, es una categoría polisémica.

Discusiones recientes ${ }^{1}$ apuntan a la necesidad de pensar en una epistemología de la gestión cultural latinoamericana, capaz de dar cuenta de tecnologías sociales y pensamiento situado sobre movilización simbólica que ha articulado, durante décadas, la organización comunitaria, la educación y comunicación popular, el activismo político, economías reproductivas, entre otras. A pesar de estos

1 Ver Carlos Yañez. Emergencias de la gestión cultural en América Latina, Manizales: Universidad Nacional de Colombia, 2014; y "Resoluciones preliminares del Primer Congreso Latinoamericano de Gestión Cultural". Santiago de Chile, del 23 al 27 de abril de 2014. vectores comunes en varias experiencias de acción cultural en este lado del continente, ¡es posible hablar de ello bajo las limitaciones de la categoría identitaria de "lo latinoamericano"? Esta pregunta no pretende ser respondida en este artículo; tampoco este texto apunta a la construcción de una homogeneidad discursiva, sino, más bien, desde un pensamiento en los límites (Hall, 2011), intentaré desestabilizar los principios "universales" que configuran a la gestión cultural, y a su vez invitaré a ubicar temporalmente su surgimiento en términos geopolíticos e históricos, tomando como eje de análisis al caso ecuatoriano.

El discurso de la gestión cultural se instaura como forma válida y legítima de accionar la cultura, con mayor fuerza, tanto en Ecuador como en otros países latinoamericanos, en los noventa. Parto de la certeza de que ninguna categoría es políticamente neutra; de ahí que, en un momento histórico de implementación y auge de políticas de ajuste estructural y neoliberales en América Latina, la gestión cultural comenzó a posicionarse en el imaginario de agentes artístico-culturales autónomos como un campo naciente de profesionalización para un oficio que aparentemente se había desarrollado de forma empírica y espontánea. Sin embargo, para cuestionar esta afirmación, basta una breve revisión histórica que identifique cómo en el país se fue configurando un potente tejido de expresiones artísticas y culturales que, entre los años sesenta y noventa, imaginó una serie de estrategias para accionar lo cultural, formas de producción, generación de sentidos críticos alrededor de la circulación de propuestas artísticas, y procesos en permanente relación con comunidades, gremios de trabajadores, estudiantes, nuevas migraciones que ocuparon nacientes barrios urbanos, organizaciones 
sociales, movimientos de izquierda, grupos asociados a la Teología de la Liberación, así como colectivos culturales que promovían el ejercicio político de la toma y uso del espacio público. Estas prácticas que tensionan relaciones de poder en el campo del arte y en la administración de las instituciones culturales públicas, operaron con autonomía política o, en algunos casos, se situaron en una posición fronteriza.

En definitiva, a partir de la década de los sesenta, artistas, trabajadores de la cultura, promotores y difusores, configuraron sus prácticas bajo el impulso que dio continuidad a la búsqueda de un espíritu nacional, y la convicción de que el desarrollo político de la clase obrera pasa por la cultura. La Revolución Cubana y el pensamiento postcolonial fueron marcos de actuación para prácticas estéticas políticas, anticapitalistas y de labor mancomunada con comunidades diversas. Las memorias del I Congreso Latinoamericano y del Caribe de Promotores y Animadores Culturales (1992), realizado en Ecuador, en 1992, evidencian que hasta ese momento las ideas que movilizaban la acción cultural a nivel local en prácticas con fuerte dimensión política y comunitaria, se asentaban en debates sobre la cultura nacional-popular, la teoría de la dependencia, el fortalecimiento de la identidad nacional frente a la dominación simbólica y la homogenización de la cultura de masas, la resistencia a la privatización, y el impulso a procesos de cambio y transformación política.

Solo entrados los noventa, se habría producido una ruptura de estos sentidos que fueron configurando un pensamiento sobre gestión cultural en el país y Latinoamérica. Si tomamos la afirmación de Irmgard Emmelhain (s/f), podríamos determinar que tras la caída del
Muro de Berlín, la reunificación alemana, y por tanto, los cambios de organización geopolítica mundial y la aplicación de políticas neoliberales y multiculturales en América Latina, ocurre una suerte de incorporación simbólica de organizaciones sociales, de mujeres, de pueblos y nacionalidades indígenas y afrodescendientes, diversidades sexuales, y también de movimientos artísticos y culturales, que hasta este entonces se declaraban anticapitalistas; es decir, a través de una serie de programas de desarrollo y ajuste estructural, estos movimientos se convierten en objeto de intervención, y se declara como su nuevo horizonte emancipatorio al emprededurismo (Emmelhain, s/f). Tal como ocurrió con otros movimientos sociales de la región, en el campo artístico cultural, a través de la gestión como tecnología de gobierno _ es decir, como medio técnico de gobierno de la conducta para lograr comportamientos fijados en unos objetivos previos (Castro Gómez, 2010) , la implementación de políticas de ajuste estructural con la agenda global de desarrollo, dirigida por organismos multilaterales de financiamiento, el concepto de "desarrollo humano" promovido por el PNUD, entre otros, intentaron sobreponerse a tecnologías sociales de acción cultural existentes, instaurando órdenes gerenciales estructurados bajo nuevos preceptos tecnocráticos y administrativos: el proyecto de gestión, la generación de indicadores, sistemas de diagnóstico, planificación, evaluación, sostenibilidad, etc.

Como medio técnico, el relato de la gestión cultural debía ser interiorizado en las poblaciones sobre las que había que intervenir; para ello, programas de gobierno, de educación y de ayuda al desarrollo, usaron dispositivos subjetivos: la cultura como estrategia de lucha contra la pobreza y mejoramiento de 
las condiciones sociales, lo cual implicaría el deseo de progreso, de "una vida mejor" -es decir, la legitimación de la cultura basada en su utilidad, en su capacidad de resolver problemas aparentemente ubicados fuera de la esfera cultural, y por tanto, a comprender que los impactos de inversión en cultura se deberían reflejar en otras áreas sociales- (Yúdice, 2006, 15); el desarrollo humano y el autogobierno de sí mismo, por tanto, la capacidad de los sujetos de generar emprendimientos; y en diálogo con las dos anteriores, la necesidad de profesionalización de agentes culturales como gestores, con el fin de contrarrestar su baja productividad y lograr su inserción en el mercado.

Es claro que existe una evidente intencionalidad que vincula estrechamente las políticas neoliberales en los noventa, la interiorización del discurso de la gestión cultural en agentes del campo artístico, y la necesidad de fomentar emprendimientos, que, por supuesto, debían estar administrados por gestores capacitados en habilidades gerenciales. Habría que preguntarse, desde entonces, qué flujos epistemológicos resisten y desestabilizan el orden hegemónico de la gestión cultural y sus imperativos interiorizados en esta década, $\mathrm{y}$ cuáles han sido negociados $\mathrm{y}$ apropiados para el agenciamiento de procesos actuales. Lo cierto es que, en ese periodo, ocurre un giro hacia la institucionalización y tecnocratización de los movimientos sociales y con ello de ciertos agentes y colectivos artístico-culturales que apuntaban con fuerza a una gestión cultural transformadora y muy ligada a lo comunitario.

La asociación de la gestión cultural con un imaginario de profesionalización fue un mecanismo útil para esta institucionalización. Este proceso inició en Ecuador a fines de los ochenta con seminarios de capacitación para personal de instituciones públicas culturales con asesoría de organismos internacionales, especialmente $\mathrm{UNESCO}^{2}$. En estos espacios, la circulación de herramientas administrativas como planificar, programar, ejecutar y evaluar, estructuran los programas formativos dirigidos a los funcionarios. Hay que recordar que la necesidad de planificación imperó en el país a partir de los setenta, cuando durante los gobiernos militares se buscó consolidar una modernización del Estado, que se expandió al campo cultural desde fines de esta década, y tuvo como centro a la idea de desarrollo cultural y la exaltación de lo nacional: "el Estado comienza a delinear una idea y administración de la cultura" (Sánchez Parga, 1988, 40). A fines de los setenta, se crea el Instituto Nacional de Patrimonio Cultural, se promulga la Ley de Patrimonio Cultural y se otorgan competencias culturales al Banco Central; en 1979, con el retorno a la democracia, la cultura se incorpora al Plan Nacional de Desarrollo 1980-1984, se aprueba la Ley de Cultura de 1984 y con ella el Fondo Nacional de Cultura, se crean instituciones públicas del gobierno como la Subsecretaría de Cultura del Ministerio de Educación y Cultura en 1981, se inaugura nueva infraestructura cultural pública, entre otros. Por supuesto, este incremento del aparataje público, demandó la formación en gestión cultural de los funcionarios, con el fin de que estos atendieran las necesidades administrativas de las nuevas instancias; de ahí que, especialmente desde mediados de los ochenta, estas instituciones públicas hayan solicitado ayuda internacional para capacitación técnica.

2 Ver "Cultura: Informe sobre los seminarios de formación y perfeccionamiento de personal para acciones culturales en Quito", Ministerio de Educación y Cultura, 1987. 
Por otra parte, este proceso de modernización que arranca en los setenta, implicó también la creciente importación de bienes y ofertas culturales, que, junto a la ampliación de la clase media y el cambio de consumos culturales, traerá como consecuencia en la década siguiente "la aparición de determinadas mediaciones, que se constituyen tanto de los mismos actores sociales como desde las instituciones públicas" (Sánchez Parga, 1988, 69). "Las mediaciones" a las que hace referencia el autor citado corresponden a la emergencia de perfiles asociados con la gestión cultural o lo que Mónica Lacarrieu $(2002,11)$ denomina nuevos intermediarios culturales, que surgen debido al cambio de las relaciones entre cultura, economía y política, y en un contexto de nuevas alianzas público privadas que requieren de habilidades relacionadas a la comunicación, el entretenimiento, la publicidad, el marketing, entre otros.

En conclusión, el lenguaje de la planificación, del proyecto, de la sostenibilidad, comenzó a tonarse dominante en el país, en un primer momento, en los aparatos culturales de Estado, especialmente desde el periodo de gobierno de Febres Cordero (19841988); se trataba, entonces, de un lenguaje homogéneo interiorizado a través de programas de cooperación y formación técnica, al que los agentes artísticos y culturales autónomos y "empíricos" debían comenzar a adecuarse para ser considerados interlocutores válidos o "beneficiarios" de recursos públicos de cultura.

Este lenguaje que responden a procesos de modernización estatal, también tiene profundas vinculaciones con las políticas neoliberales, las intenciones de privatizar ciertas actividades públicas culturales y el impulso a la generación de emprendimientos a fines de los ochenta. La política cultural del gobierno de Febres Cordero hizo eco a la de Thatcher, que consistía en programas de privatización en lo cultural, recortes de subvenciones públicas y la disminución de fondos o subvenciones no reembolsables, implementando un sistema de "ayudas con retorno" o créditos blandos, que buscaban la multiplicación de emprendimientos. En una suerte de política paralela, el gobierno de Febres Cordero siguió esta línea y creó la entidad crediticia Foncultura, un sistema de préstamos reembolsables y no reembolsables -denominada el banco de la cultura- "con el fin de propiciar el autofinanciamiento, otorgar créditos y financiar proyectos culturales." (Astudillo, 1998, 65). Estos años se caracterizaron por recortes presupuestarios al sector educativo y cultural, el estancamiento de las leyes del libro y del cine, y la desmovilización constante de grupos sociales organizados.

Mientras esto ocurría en la esfera institucional, agentes culturales se mostraban ajenos aún al discurso de la gestión y sus herramientas técnicas, y continuaron generando prácticas paralelas de movilización simbólica que se situaban al margen de este nuevo paradigma: "En este sentido países como el Ecuador, que carecen de condiciones para implementar y desarrollar una administración empresarial de la cultura, no solo se mostrarán refractarios a las propuestas neoliberales y de privatización de los procesos y productos culturales, sino que desde la misma sociedad continuarán generándose formas de cultura, algunas inéditas y de larga duración otras, reivindicaciones recientes y otras más antiguas, que se sustraen a un proyecto como el neo-liberal, a su gestión de empresa y a sus posibilidades de mercantilización". (Sánchez Parga, 1988, 161). 
Resulta fundamental recordar que, habría subsumido denominaciones anteriores, previo al boom de la gestión cultural en los noventa, en décadas anteriores se utilizaron, en el país y en la región, otras formas de nombrar al oficio de accionar la cultura: animador y promotor cultural, administrador y gerente cultural, y trabajador cultural. Otras denominaciones como mediadores, ingenieros o científicos culturales no tuvieron eco en la región (De Zubiría, Abello et al, 1998, 19). Habría que analizar, entonces, desde dónde se enunciaron la animación y promoción cultural que fueron las más importantes en nuestro contexto, cómo se resignificaron en lo local y cómo fueron colocadas en agendas públicas y de agentes y colectivos artísticos y culturales; así también, es importante reflexionar cómo cada una reemplazó o convivió con otras categorías que definieron la acción cultural, en qué contexto histórico y qué política cultural determinó su uso. Por otro lado, la definición de gerentes y administradores culturales se utilizó sobre todo en el sector público, con vigencia especialmente en los ochenta, tras varios programas de formación y capacitación técnica internacional bajo esta denominación.

El discurso de la gestión cultural cobró vigencia a través de distintos mecanismos de "profesionalización" (becas, cursos, talleres, promovidos por UNESCO, OEI, AECID), sustentados, como ya se mencionó, en el argumento de que las prácticas de acción cultural de agentes autónomos, existentes hasta ese momento (animadores, promotores, trabajadores culturales), eran espontáneas, empíricas e improvisadas, $y$, en definitiva, necesitaban profesionalizarse. $\mathrm{Al}$ respecto se han manejado también otras hipótesis: que la gestión cultural, como por generación espontánea, sin que responda a proyecto político alguno, sin modificaciones relevantes, compartiendo principios y criterios. Otra postura promueve la necesidad de mantener denominaciones anteriores, apelando a las diferencias de prácticas y discursos de otras formas de movilización simbólica frente a la "gestión", categoría que "en el ámbito cultural termina borrando las fronteras entre las actividades económicas y los procesos culturales (...)" (De Zubiría, Abello et al, 1998, 20). Autores como Jesús Martín Barbero o García Canclini sitúan a la gestión cultural y sus instrumentos como una respuesta a las transformaciones complejas que ha experimentado la dimensión cultural a partir de los noventa, afirmando que "lo gestionable en la cultura sólo puede entenderse a la luz de lo no gestionable, ya que la libertad, la autonomía la independencia de los procesos culturales no son gestionables" (De Zubiría, Abello et al, 1998, 21). Finalmente, en este artículo, planteo la hipótesis de la gestión cultural como estrategia de despolitización de prácticas artísticas y culturales de dimensión comunitaria y política, a través de la necesidad de "profesionalización" como mecanismo de interiorización de esta denominación emergente; también, apoyo firmemente la necesidad de discutir una epistemología de la gestión cultural latinoamericana, y apelo, asimismo, a la necesidad de leer a la gestión cultural como una categoría política.

En conclusión, si, por un lado, la gestión cultural constituyó sobre todo una herramienta que requería ser interiorizada para la generación y administración de emprendimientos como nuevo horizonte emancipatorio de movimientos artísticos y culturales (Irmgard Emmelhain), fue instituida inicialmente en Ecuador como un instrumento para modernizar los procesos 
estatales administrativos en cultura. Lo cierto que es que, entrados los 2000, varias prácticas autónomas y sus principios de organización de lo cultural y formas de producción de valor, intentaron ser homogenizadas por el orden establecido de la gestión cultural y sus principios "universales". Estas tecnologías sociales, con permanencias actuales, han sido escasamente investigadas y problematizadas; así también, han tenido poca o nula incidencia en la administración pública cultural y en espacios de enseñanza de esta disciplina en construcción. Lejos de un escencialismo e idealización de prácticas sociales y culturales, resulta urgente un desplazamiento político-epistémico de la lógica tecnocrática e instrumental de la gestión cultural imperante y un desafío a la narrativa construida en un momento histórico específico.

\section{Bibliografía}

Astudillo, J. "Ecuador: La gestión cultural en las últimas décadas". En La Gestión Cultural. Memorias del Curso de Postgrado en Gestión Cultural, Cuenca: Universidad de Cuenca, Consejo Nacional de Cultura, 1998.

Castro Gómez, S. Historia de la gubernamentalidad. Razón de Estado, liberalismo y neoliberalismo en Michel Foucault, Bogotá: Siglo del Hombre Editores, Universidad Javeriana, Universidad Santo Tomás, 2010.

Consejo Nacional de Cultura. I Congreso Latinoamericano y del Caribe de Promotores y Animadores Culturales, Quito: Conejo Nacional de Cultura, 1992.

De Zubiría Samper, S.; Abello, I.; Tabares, M. Cuadernos Cultura I OEI. Conceptos básicos en administración y gestión cultural, Madrid: OEI, 1998, 9-22.

Emmelhain, I. "Formas de politización del arte en la "post-política"”, s/f. En http://www. ramona.org.ar/node/59794
Hall, S. La cultura y el poder: Conversaciones sobre los cultural studies. Buenos Aires: Amorrortu, 2011.

Lacarrieu, M. "La plaza y la caverna: dilemas contemporáneos en la gestión cultural. En $L a$ (indi) gestión cultural. Una cartografía de los procesos culturales contemporáneos. Argentina: Ediciones Ciccus y La Crujía, 2002.

Ministerio de Educación y Cultura del Ecuador. Cultura: Informe sobre los seminarios de formación y perfeccionamiento de personal para acciones culturales en Quito. Ecuador: Ministerio de Educación y Cultura, 1987.

"Resoluciones preliminares del Primer Congreso Latinoamericano de Gestión Cultural". Santiago de Chile, del 23 al 27 de abril de 2014. En: http://redlgc.ning. $\mathrm{com} /$ notes/Resoluciones_preliminares_ Primer_Congreso_Latinoamericano_de_ Gesti\%C3\%B3n_Cultural

Richard, N. "Localidades del saber: ubicación, contextos y traducción”. En Colonialidad/ decolonialidad del poder/saber. Miradas desde el Sur, Valdivia: Ediciones Universidad Austral de Chile, 2012.

Sánchez-Parga, J. Actores y discursos culturales Ecuador: 1972-88, Quito: Centro Andino de Acción Popular, 1988.

Soto, M. "Apuntes para la definición de los nuevos perfiles de profesionales en gestión cultural y una agenda de acciones para el desarrollo de los programas de formación de la región latinoamericana". En Campus Euroamericano de Cooperación Cultural 5, 2007, mayo 8-12. Almada. Madrid: OEI, 2008, 209214.

Yáñez, C. "Enfoques y tendencias de la Gestión Cultural en América Latina”. En Emergencias de la gestión cultural en América Latina. Manizales: Universidad Nacional de Colombia, 2014.

YÚDICE, G. El recurso de la cultura. Usos de la cultura en la era global, Barcelona: Gedisa, 2006. 